\title{
Quantum States as Objective Informational Bridges
}

\author{
Richard Healey \\ University of Arizona
}

February 1st, 2015

\begin{abstract}
A quantum state represents neither properties of a physical system nor anyone's knowledge of its properties. The important question is not what quantum states represent but how they are used - as informational bridges. Knowing about some physical situations (its backing conditions), an agent may assign a quantum state to form expectations about other possible physical situations (its advice conditions). Quantum states are objective: only expectations based on correct state assignments are generally reliable. If a quantum state represents anything, it is the objective probabilistic relations between its backing conditions and its advice conditions. This paper offers an account of quantum states and their function as informational bridges, in quantum teleportation and elsewhere.
\end{abstract}


the quantum state of a Qbit or a collection of Qbits is not a property carried by those Qbits, but a way of concisely summarizing everything we know that has happened to them, to enable us to make statistical predictions about the information we might then be able to extract from them. (N. David Mermin [2007, p. 109])

\section{Introduction}

In his recent extended review article, Matt Leifer [2014] classifies the pragmatist view of quantum theory sketched in my [2012a] as $\psi$-epistemic rather than $\psi$-ontic while acknowledging that the $\psi$-ontology theorems he discusses do not apply to it. But as he draws the $\psi$-ontic/ $\psi$-epistemic distinction in his introduction, that pragmatist view counts as neither $\psi$-epistemic nor $\psi$-ontic. It does not view $|\psi\rangle$ as describing some independently existing property of the system to which it is assigned, but nor does it take a quantum state to exist only in the minds and calculations of quantum theorists. This paper is intended to clarify my [2012a] view of the quantum state, and to show how $|\psi\rangle$ refers to something that objectively exists in the world, independently of any observer or agent, and would still exist if all intelligent beings were suddenly wiped out from the universe - which is how Leifer characterizes an ontic state.

A quantum state (given by a wave-function, vector or density operator) represents neither properties of a physical system to which it is assigned, nor anyone's knowledge of its properties. The important question is not what quantum states represent but how they are used - as informational bridges. Knowing about some physical situations (its backing conditions), an agent may assign a quantum state to form expectations about certain other possible physical situations (its advice conditions). Quantum states are objective: only expectations based on correct state assignments are generally reliable. But a quantum state represents neither its backing conditions nor its advice conditions.

Since its main function is to provide information on what advice conditions to expect given prevailing backing conditions, it may be said to represent probabilistic relations between backing conditions and advice conditions. These probabilistic relations are objective: they would exist in a world without agents, as long as that world featured patterns of statistical regularity that were sufficiently stable to be modeled by Born probabilities of the values of physical magnitudes that specify what I have called advice conditions. We can use quantum theory successfully because (or at least in so far as) they do exist in our world.

As physically situated, and so epistemically limited, a user of quantum theory can assign quantum states on the basis of what that agent is in a position to know, in order to form reasonable expectations about what that agent is not in a position to know. That is how quantum states function as informational bridges. A quantum state provides a sturdy informational bridge only if it would be the right state to assign for any agent in that physical, and therefore epistemic, situation - only if it refers to the actual probabilistic relations between 
its accessible backing conditions and inaccessible advice conditions.

Here is the structure of the rest of the paper. By distinguishing between quantum mathematical models and their physical applications section 2 shows how a precise formulation of quantum theory may be given without using terms like 'measurement', 'observation', 'information' or 'agent'. Any account of how the theory is applied must mention agents who may apply it: agents are (potential) users of quantum models - they are not mentioned in those models. Any application of a quantum model is perspectival - it is from the perspective of a hypothetical, physically situated, agent. So to say how a model of quantum theory is applied we need a rough physical characterization of this situation as what I will call an agent-situation. Section 2 supplies this and clarifies the notions of backing conditions and advice conditions. A correct quantum state assignment is relative to an agent-situation because these conditions are a function of that situation. Section 3 shows the worth of these ideas in explaining how quantum theory works in some applications that have been thought to raise problems of "wave collapse" and causal anomalies. Chris Timpson [2013] used his analysis

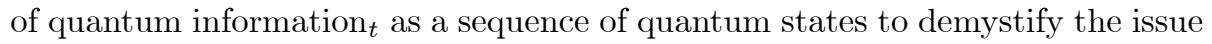
of information flow in quantum teleportation. But there is more to be said if a quantum state itself functions as an informational bridge: I say it in section 4. The paper concludes by summarizing its main points and relating them to recent work in quantum foundations.

\section{Quantum states: Objective but relational}

In discussing quantum states we should start by distinguishing the state itself from any of its mathematical representatives, including $|\Psi\rangle$ (or a wave-function representation such as $\left.\psi\left(\mathbf{r}_{1}, \ldots, \mathbf{r}_{n}\right)\right)$, $\hat{\rho}$, or (as in AQFT) $\omega: \mathcal{A} \longrightarrow \mathbb{C}$. As a mathematical object, none of these representatives of a quantum state exists in the physical world: what is at issue is the nature and function of the quantum state such objects purport to represent. But that issue is best approached indirectly, by studying the role of a mathematical representative of a quantum state in an application of a mathematical model of quantum theory of which it is one element.

Such models take different forms in different kinds of quantum theory, but since the same issue arises for each I'll consider only models of nonrelativistic quantum mechanics of the form $\Theta=\langle\mathcal{H}, T, \Psi, \mathcal{A}, \mu\rangle$. In an application, the interval of real numbers $T$ will represent an interval of time while $t \in T$ represents a moment in that interval. Each operator in $\mathcal{A}$ is linear: in an application a self-adjoint operator $\hat{A}$ will correspond uniquely to a dynamical variable $A$. The self-adjoint operator $\hat{H}$ corresponding to energy is associated with a family of unitary operators $\hat{U}_{t}=\exp i \hat{H} t$ that define the possible trajectories of $|\Psi(t)\rangle$ (in the Schrödinger picture). The measure $\mu$ assigns, at each $t \in T$, a number in the interval $[0,1]$ to each subspace $\mathcal{K}$ of $\mathcal{H}$ such that $\mu(\mathcal{H})=1$, $\mu(\varnothing)=0$ and if $\mathcal{K}_{i} \perp \mathcal{K}_{j}$ for $i \neq j$ then $\mu(\mathcal{L})=\sum_{i} \mu\left(\mathcal{K}_{i}\right)$, where $\mathcal{L}=\vee_{i} \mathcal{K}_{i}$ is the span of the $\mathcal{K}_{i}$. In an application some values of $\mu$ may yield Born probabilities. 
To apply a model of the form $\Theta$ one first chooses the dimension of a Hilbert space $\mathcal{H}$ in which to represent a quantum state $\Psi$ of a physical system $s$ over a period of time $T$ and a particular $\hat{H}$ on $\mathcal{H}$ : then one assigns a particular initial state $\left|\Psi\left(t_{0}\right)\right\rangle$ to $s$. This picks out a unique trajectory $|\Psi(t)\rangle$ and so a particular state $\left|\Psi\left(t_{1}\right)\right\rangle$ at a later time $t_{1} \in T$. Next one chooses a pairwisecommuting family $\mathcal{F}=\{\hat{A}, \hat{B}, \ldots\}$ of self-adjoint operators on $\mathcal{H}$ corresponding to dynamical variables $A, B, \ldots$ respectively. One can now apply the Born rule in the form

$$
\begin{gathered}
\operatorname{Pr}([A \in \Gamma] \&[B \in \Delta] \& \ldots)=\left\langle\Psi\left(t_{1}\right)\left|P^{A}(\Gamma) . P^{B}(\Delta) \ldots\right| \Psi\left(t_{1}\right)\right\rangle \\
=\mu_{\Psi\left(t_{1}\right)}\left(\mathcal{M}_{\Gamma}^{A} \wedge \mathcal{M}_{\Delta}^{B} \wedge \ldots\right)
\end{gathered}
$$

where the elements $P^{A}(\Gamma), P^{B}(\Delta), .$. of the spectral measures of $\hat{A}, \hat{B}, \ldots$ project onto subspaces $\mathcal{M}_{\Gamma}^{A}, \mathcal{M}_{\Delta}^{B}, \ldots$ respectively of $\mathcal{H}$. This yields a joint probability distribution for the values of dynamical variables $A, B, \ldots$ on $s$ at $t_{1}$.

While nowhere explicitly mentioning measurement or preparation, this familiar account may seem implicitly to depend on these notions. Doesn't assignment of a particular state to a system at $t_{0}$ require knowledge of how this has been prepared? Don't Born probabilities refer only to the outcome of a joint measurement at $t_{1}$ of a compatible family of dynamical variables defined by the measurement context? And doesn't the applicability of the model at time $t_{1}$ presuppose that no measurement occurred between $t_{0}$ and $t_{1}$ ?

In fact one can say what warrants assignment of a quantum state and what circumstances license application of the Born rule without mentioning preparation or measurement. In each case it is simply the prevailing physical conditions that supply the necessary warrant, whether or not anyone seeks to exploit these to prepare the system's state or make a measurement on it. In the first case I call these backing conditions, since when they obtain they back assignment of a particular quantum state. In the second case environmental conditions determine when and how the Born rule may legitimately be applied to provide reliable advice about the values of certain dynamical variables, in the form of what I call advice conditions.

I headed this paper with a quote from David Mermin in which he characterizes a quantum state of a system as a way of concisely summarizing everything we know that has happened to it. ${ }^{1}$ The knowledge of which he speaks is knowledge of backing conditions. Knowing a state's backing conditions, one is justified in assigning that state: but one would be warranted in assigning the state whether or not one knew these conditions, just as a test result may warrant a diagnosis whether or not the doctor knows about it. I'll give several examples of backing conditions later. For now it is important only that they may be stated in purely physical language, with no talk of observers or agents and their measuring or preparing activities. But nor does this statement involve talk of quantum states, operators or Hilbert space measures: such talk is confined to the mathematical model being applied.

\footnotetext{
${ }^{1}$ In two earlier papers (Mermin [2002], Brun, T., Finkelstein, J. and Mermin, N.D. [2002]) he had raised and answered the question "Whose knowledge?".
} 
Advice conditions are stated in a more restricted physical language because of how they figure in the Born rule. When legitimately applied, that rule yields probabilities for what I'll call magnitude claims: The value of dynamical variable $M$ on physical system s lies in $\Delta$ is a canonical magnitude claim which I'll write as $M_{s} \in \Delta$. Magnitude claims are used to state advice conditionsso-called because they are the topic of the Born rule's probabilistic advice. But that advice is necessarily selective. The Born rule yields joint probabilities only for magnitude claims corresponding to commuting projection operators, and Gleason's [1957] theorem and other results show that these typically cannot all be recovered as marginals of any underlying joint distribution.

Restrictions on use of the Born rule are needed to ensure its consistent application to a system, but these arise naturally from the physical circumstances in which that system finds itself. $\Theta$ provides an adequate quantum model of a system $s$ only while $s$ remains isolated from interaction with other quantum systems: If $s$ is subjected to such interactions, this can also be modeled by the unitary evolution of a joint quantum state in an expanded Hilbert space. In various models of decoherence this extremely rapidly and essentially irreversibly results in the delocalization of phase of the quantum state of $s$ and/or a system $\alpha$ with which it interacts into the wider environment, thereby picking out a preferred "pointer basis" of orthogonal subspaces in their Hilbert space(s).

The Born rule may be legitimately applied to $s$ only when such decoherence has occurred, and then only to those privileged magnitude claims corresponding to projection operators onto subspaces in the relevant pointer basis. $^{2}$ Such decoherence is never perfect, and nor is a "pointer basis" precisely determined and perfectly constant. But the advice provided by the Born rule concerning only magnitude claims privileged by pointer bases in the same narrow neighborhood will be consistent and typically prove reliable: the corresponding advice conditions will typically obtain with relative frequencies closely corresponding to their Born rule probabilities.

You and I may know different things about what has happened to a quantum system. Should we then assign the system different quantum states? If you have simply noticed something I missed, or I have forgotten something you remember, then one of us has not made use of all the available information: our quantum state assignment should be based on all the information to which we have access. But the information to which an agent has access depends on that agent's physical situation.

A single agent gains access to additional information merely with the passage of time, while spatially separated agents have access to different information just because they are in different places. It is not only spatiotemporal location that imposes physical limits on one's knowledge of what has happened to a system. Acquisition of observational knowledge depends on the presence of physical channels capable of conveying that knowledge to the observer, which is why it is so hard to observe dark matter in distant galaxies. The upshot is that when an agent assigns a quantum state the conditions backing that assignment

${ }^{2}$ For further details, see my [2012b]. 
are a function of the agent's physical situation.

We often apply quantum theory not to the actual world but to some simplified or otherwise modified variant of it - to a merely possible world. Clearly the agent applying the model is not located in that merely possible world. But even such applications are from the perspective of some merely possible agent in that world - the perspective of what I will call an agent-situation. All applications of quantum theory are from the perspective of a physically-characterized agent-situation. Applications to merely possible worlds make it particularly clear that no agent need occupy such an agent-situation.

An agent-situation is characterized, in part, by a space-time region marking the momentary location of a hypothetical agent. It is common to idealize the histories of observers by timelike curves in a relativistic space-time - their world-lines. In this idealization the space-time location of an agent-situation would be a point $p$ on such a curve. Since no physical processes can convey information faster than light, no agent at $p$ would have observational access to anything outside the past light cone of $p$. So the backing conditions for a quantum state assignment relative to $p$ lie in (or on) the past light cone of $p$.

A quantum state assignment to a system relative to $p$ will be a function of everything in the past light cone of $p$ (though much of what happens there will prove irrelevant). But it is important to notice that quantum state assignments relative to $p, q$ may differ: conditions obtaining in the past light cone of $p$ may back a different assignment than conditions obtaining in the past light cone of $q$. Since every quantum state assignment is relative to an agent-situation it is misleading to speak of a system's being in a quantum state, as if this expressed a property of that system rather than a relation to the agent-situation from whose perspective it is assigned.

\section{Some applications}

Before moving on to its application to the phenomenon of quantum teleportation, I'll consider two illustrative examples of this account of quantum states as objective informational bridges - violation of Bell inequalities and delayed choice entanglement-swapping.

Quantum theory correctly predicts the violations of Bell inequalities that have been observed in experiments involving space-like separated measurements of linear polarization on polarization-entangled photon pairs (EPR-Bohm pairs) (Weihs et al. [1998]). Bell [2004] used these predictions to argue that quantum theory is not a locally causal theory. Maudlin [2011] argued further that space-like (superluminal) influences must be present in experiments verifying them: Price [1996], on the other hand, suggested their results might be explained by retrocausal influences. One way for a measurement on one photon in an EPR-Bohm pair to influence the result of a spacelike separated measurement on the other photon would be through "collapsing its quantum state" - just the kind of "spooky" action at a distance Einstein rejected. Any such instantaneous collapse would threaten to reintroduce an absolute notion of 
simultaneity into a relativistic space-time from which it had been successfully excised.

In fact quantum theory may be applied to explain EPR-Bohm correlations with no superluminal or retro- causation and no physical quantum state reduction. ${ }^{3}$ The relativization of quantum states to agent-situation is key to this explanation. An entangled state $\Phi^{+}=1 / \sqrt{ } 2(|H H\rangle+|V V\rangle)$ may be correctly assigned to the pair relative to an agent-situation with location $p$ in the past light cone of either of the space-like separated polarization measurement events: its backing conditions (in the past light cone of $p$ ) include whatever physical conditions the experimenters used to prepare that state, such as parametric down-conversion of laser light by passage through a non-linear crystal.

Because of decoherence at the detectors, the Born rule is legitimately applied to this state to yield an equal chance of either outcome of any linear polarization measurement at either detector, but chance $\cos ^{2} \angle a b$ that the two detectors will yield the same outcome for linear polarization measurements with respect to axes inclined at an angle $\angle a b$ to one another. It is important to stress that these chances are also relative to agent-situation with location $p$. For anyone who accepts quantum theory, they give the objectively correct advice to one located at $p$ about how firmly to believe the corresponding outcome(s) will be recorded. Thus state $\Phi^{+}$acts as an informational bridge between its backing conditions and its advice conditions by enabling anyone informed of its backing conditions to form reliable expectations concerning it advice conditions.

Consider instead an agent situation with location $q$ in the future light cone of recording event $\mathbf{1}$ but not of recording event $\mathbf{2}$. Relative to $q$, the correct quantum polarization state to assign to the distant photon in the past light cone of $\mathbf{2}$ depends on the outcome at 1: suppose it is $V_{a}$, where linear polarization at $\mathbf{1}$ was measured with respect to axis $a$. Then $\left|V_{a}\right\rangle$ is the correct quantum state to assign to the distant photon, relative to agent situation with location $q$. This is not because "the" quantum state of the pair has collapsed: the correct quantum state to assign relative to agent-situation with location $p$ is still $\frac{1}{2} \hat{1}$ the reduced state of $\Phi^{+}$. The correct state relative to agent situation with location $q$ is $\left|V_{a}\right\rangle$ because the outcome at $\mathbf{1}$ is in the past light cone of $q$, and so counts as an additional accessible backing condition determining the assignment of a quantum state relative to $q$. Relative to agent situation with location $q$ the chance of outcome $V_{b}$ at $\mathbf{2}$ is $\cos ^{2} \angle a b$, as follows from the legitimate application of the Born rule to state $\left|V_{a}\right\rangle$. Here it is state $\left|V_{a}\right\rangle$ that acts as an informational bridge between its (augmented) backing conditions and its advice conditions.

These applications of quantum theory explain the patterns of correlation that are taken to violate CHSH inequalities by showing that they were to be expected and what they depend on. Both individual outcome events and the event of their joint occurrence depend causally on the physical conditions backing assignment of $\Phi^{+}$since an agent could affect the chances of these events by modifying those backing conditions. But there is no causal dependence of one outcome event on the other, since no-one who accepts quantum theory

${ }^{3}$ For a fuller account see my [forthcoming]. 
can countenance the possibility of an agent's modifying either outcome while keeping fixed both detectors' settings and the conditions backing $\Phi^{+}$. Nor do the detector settings have any retrocausal influence on events acknowledged by quantum theory.

Now consider delayed-choice entanglement-swapping. Suppose two EPRBohm photon pairs $\langle 1,2\rangle,\langle 3,4\rangle$ are independently prepared in conditions backing assignment of Bell states $\Psi_{12}^{-}, \Psi_{34}^{-}$

$$
\Psi^{-}=1 / \sqrt{ } 2(|H V\rangle-|V H\rangle)
$$

First assume a Bell-state measurement is conducted on $\langle 2,3\rangle$, and the result noted. Conditional on a particular result, one of four different entangled states may then be assigned to the pair $\langle 1,4\rangle$. This is entanglement-swapping. ${ }^{4}$ The particular entangled state of $\langle 1,4\rangle$ may be verified by quantum tomography in the usual way, and violation of Bell inequalities demonstrated. Alternatively, assume the linear polarizations of each of 2,3 are measured independently (instead of the Bell-state measurement). No matter what the outcomes of the measurements on 2,3, measurements of linear polarization on 1,4 will reveal no correlations: since the states then assigned to 1, 4 are not entangled their joint probability distribution will factorize.

So far I have said nothing about when and where the measurements on 1,4 occur, relative to those on 2,3 . In a standard case of entanglementswapping, the Bell-state measurement $B_{23}$ on 2,3 occurs time-like earlier than each of the measurements $M_{1}, M_{4}$ on 1,4. But the correlations between the outcomes of measurements $M_{1}, M_{4}$ conditional on a particular (joint) outcome of a Bell-state measurement $B_{23}$ do not depend on the space-time intervals $I\left(B_{23}, M_{1}\right), I\left(B_{23}, M_{4}\right)$. If $B_{23}$ occurs timelike later than $M_{1}, M_{4}$ then we have an apparent case of "retrocausal entanglement swapping". Since the choice of whether to perform $B_{23}$ or independent polarization measurements $M_{2}, M_{3}$ may be made at random after photons 1,4 have been absorbed into their detectors, this has also been called delayed choice entanglement-swapping. ${ }^{5}$

Relative to an agent-situation located just in the overlap of the future light cones of their production events, the correct initial polarization state $\Psi$ to assign to the photons is

$$
\left|\Psi_{12}^{-}\right\rangle\left|\Psi_{34}^{-}\right\rangle=1 / 2\left(\left|\Psi_{14}^{+}\right\rangle\left|\Psi_{23}^{+}\right\rangle-\left|\Psi_{14}^{-}\right\rangle\left|\Psi_{23}^{-}\right\rangle-\left|\Phi_{14}^{+}\right\rangle\left|\Phi_{23}^{+}\right\rangle+\left|\Phi_{14}^{-}\right\rangle\left|\Phi_{23}^{-}\right\rangle .\right.
$$

Analysis of the actual experimental setup of Ma et al. [2013] shows that cases (i) in which $B_{23}$ records one photon as detected to each side of the beam splitter (with the same polarization) have non-zero Born probability only from the fourth term in (3), while cases (ii) in which $B_{23}$ records both photons as detected to the same side of the beam splitter (with opposite polarizations) have non zero Born probability only from the third term in (3). What would be the correct state to assign to the remaining photons, relative to an agent-situation

\footnotetext{
${ }^{4}$ By adding a classical channel it may be used to teleport entanglement of a Qbit.

${ }^{5}$ By Peres [2000]. This has been realized experimentally by Ma et al. [2013].
} 
located just in the future light cone of $B_{23}$ ? In case (i) the corresponding $\langle 1,4\rangle$ pair should be assigned the quantum state $\Phi_{14}^{-}$, while in case (ii) the corresponding pair should be assigned $\Phi_{14}^{+}$. These would be the correct state assignments irrespective of the space-time intervals $I\left(B_{23}, M_{1}\right), I\left(B_{23}, M_{4}\right)$.

A recent paper (Egg [2013, p.1133]) rejects that conclusion as follows (I have changed his notation to conform to mine):

The Bell measurement on the $\langle 2,3\rangle$ pair allows us to sort the $\langle 1,4\rangle$ pairs into four subensembles corresponding to the four Bell states. Without delayed choice, this has physical significance, because each $\langle 1,4\rangle$ pair really is in such a state after the $\langle 2,3\rangle$ measurement $\left[B_{23}\right]$. But if the $\langle 1,4\rangle$ measurements $\left[M_{1}, M_{4}\right]$ precede $\left[B_{23}\right]$, the $\langle 1,4\rangle$ pair never is in any of these states. ... Therefore, far from being committed to any indeterminacy about entanglement (or any backward-in-time influences) a realistic view of the quantum state yields a perfectly clear assessment of what happens in entanglement-swapping: If $\left[B_{23}\right]$ occurs at a time the complete system is still in state $\Psi$, it confers entanglement on the $\langle 1,4\rangle$ pair, if it occurs at a later time it does not.

In section 2 I warned against talk of a system's being in a quantum state (as opposed to being assigned the correct quantum state relative to a particular agent-situation). We can see how this leads to problems by reflecting on a case of entanglement-swapping in which the intervals $I\left(B_{23}, M_{1}\right), I\left(B_{23}, M_{4}\right)$ are space-like. In this case the distinction to which Egg appeals has no invariant significance: whether $M_{1}$ or $M_{4}$ precedes $B_{23}$ depends on an arbitrary choice of reference frame. Egg is not unaware of this problem: he admits (p.1130) that his "reality-of-states" view should take state reduction seriously, so that the change of description on "measurement" corresponds to a real physical change. His "solution" ( footnote 7, p.1130) is to appeal to a definite (although undetectable) temporal ordering between any two events - a preferred foliation of space-time such as that required by alternative theories including Bohmian mechanics.

But there is no physical process of state reduction, and no need to appeal to any undetectable space-time foliation to understand entanglement-swapping or violation of Bell inequalities. The reassignment of a quantum state associated with relativizing it to a different agent-situation is not a physical process. It is typically required because additional backing conditions are accessible from the second agent-situation. The discussion of EPR-Bohm correlations provided an example: $\left|V_{a}\right\rangle$ is the correct quantum state to assign to the distant photon relative to agent-situation $q$ while the correct quantum state to assign relative to agent-situation with location $p$ is $\frac{1}{2} \hat{1}$ : each of these quantum states is objective, but there is no question as to which quantum state the distant photon "is in".

When an entangled state is assigned to the pair $\langle 1,4\rangle$ conditional on the outcome of $B_{23}$ this state functions as an objective informational bridge in the usual way, irrespective of the space-time intervals $I\left(B_{23}, M_{1}\right), I\left(B_{23}, M_{4}\right)$. What distinguishes the case in which $B_{23}$ occurs invariantly later than $M_{1}, M_{4}$ 
is the fact that if appropriate physical channels were in place the outcomes of $M_{1}, M_{4}$ would be accessible from the space-time location of $B_{23}$. In that case there would be no point in using the entangled state assigned to the pair $\langle 1,4\rangle$ as an informational bridge to form expectations as to these outcomes, since knowledge as to what they were would already be available at the space-time location of $B_{23}$.

This illustrates the important point that there is more to an agentsituation than its space-time location. A physical characterization of an agent situation located just in the future light cone of $B_{23}$ specifies either the presence or the absence of physical channels connecting this location to the outcomes of $M_{1}, M_{4}$ : only if no such channels are in place is it necessary to assign an entangled state to $\langle 1,4\rangle$ conditional on the outcome of $B_{23}$, since only relative to that agent-situation does this state assignment provide the best available information concerning the outcomes of $M_{1}, M_{4}$. In a case in which $B_{23}$ occurs invariantly earlier than $M_{1}, M_{4}$, or space-like separated from them, an entangled state must always be assigned to $\langle 1,4\rangle$ relative to any agent-situation located just in the future light cone of $B_{23}$ and backed by the outcome of $B_{23}$, because no physical channels could give access to the outcomes of $M_{1}, M_{4}$ from that space-time location.

\section{Information in quantum teleportation}

Chris Timpson devotes two chapters of his [2013] to a discussion of information flow in quantum teleportation. His conclusion is largely deflationary

we have been able to replace the (needlessly) conceptually puzzling question of how the information ${ }_{t}$ gets from Alice to Bob in teleportation, with the simple, genuine question of what the physical processes involved in teleportation are. (p. 86)

He had previously proposed a helpful analysis of the technical notion of information $_{t}$ after distinguishing this from our everyday, informal notion. This

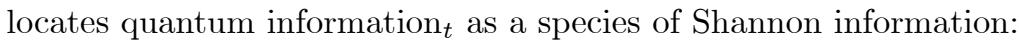

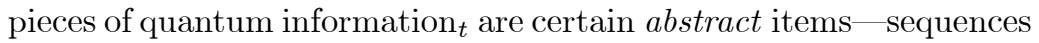
of quantum states (types)

As abstracta, pieces of quantum information ${ }_{t} \ldots$ do not themselves have a spatio-temporal location; it is their tokens (if any) which do. (p. 65)

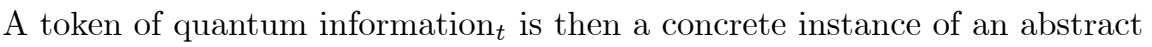
sequence of quantum states. The simplest example of a token of quantum information $_{t}$ is a single (physical) Qbit. Quantum teleportation is a physical process in which a Qbit associated with state $|\chi\rangle$ is initially located only at $A$ and a Qbit associated with state $|\chi\rangle$ is finally located at $B$ while the Qbit 
formerly located at $A$ is no longer associated with state $|\chi\rangle$. In this way the abstract piece of quantum information $t$ previously instantiated only at $A$ is now instantiated only at $B$.

Among the remarkable features of quantum teleportation is that it enables Alice to teleport an unknown quantum state to Bob. After its successful execution Alice and Bob both know that the state now associated with Bob's Qbit is the same state formerly associated with Alice's, but neither Alice nor Bob need know what this state is. A teleported state may be unknown to Alice and Bob because neither of them knows what had happened to Alice's Qbit before she got hold of it.

One can accept Timpson's helpful analysis without saying anything about the nature and function of quantum states. But suppose the quantum state of a Qbit is a way of concisely summarizing everything we know that has happened to it. If there is no-one but Alice and Bob, then the quantum state they teleport may be a way of concisely summarizing knowledge that no-one knows - an unknown known! Clearly something has gone wrong. Not even Donald Rumsfeld countenances unknown knowns. ${ }^{6}$ Correcting the errors leading to this paradoxical conclusion will prove very helpful in arriving at a clearer understanding of quantum states as objective informational bridges.

One can think of quantum teleportation as bridging an informational gap by reproducing a token of quantum information $t$ at a distant location. But the notion of information involved in calling a quantum state an informational bridge is not the technical notion (information ${ }_{t}$ ) analyzed by Timpson but the ordinary notion. A token of quantum information ${ }_{t}$ is itself an informational bridge in this ordinary sense: it bridges the gap between the state's backing conditions and its advice conditions. It is backing conditions in the past light cone of $p$ that determine the objectively correct initial quantum state to assign to Alice's Qbit, relative to $p$. What those backing conditions are depends both on the space-time location of $p$ and on the presence of physical channels capable of giving access to them from $p$.

Consider Alice's situation at space-time location $A$ as she receives her Qbit to be teleported to Bob at $B$. Reliable teleportation requires a (classical) physical channel connecting $A$ to $B$. So whatever conditions $K$ back assignment of initial state $|\chi\rangle$ to Alice's Qbit relative to an agent-situation with location $A$ apparently also back assignment of initial state $|\chi\rangle$ to Alice's Qbit relative to an agent-situation with location $B$. If no teleportation actually occurred, Bob would not have his own Qbit in state $|\chi\rangle$ at $B$, but he would appear to be in as good a position as Alice to assign state $|\chi\rangle$ to Alice's Qbit at $A$.

How could Alice not know what state to assign to her Qbit at $A$ ? Perhaps Carol agreed to prepare this Qbit for Alice at $A$ by taking a Qbit (they both agree to be) correctly assigned state $|0\rangle$ and subjecting it to a unitary transformation into state $|\chi\rangle=a|0\rangle+b|1\rangle$ without telling Alice what that transformation was. Whatever physical conditions $K^{\prime}$ involved in implementing

\footnotetext{
${ }^{6}$ Although the authors of a documentary film about him called it The Unknown Known, suggesting that he might have been one.
} 
Carol's unitary transformation backing her assignment of $|\chi\rangle$ occurred in the past light cone of $A$, but in the absence of physical channels capable of giving an agent at $A$ access to $K^{\prime}$ those conditions would not back assignment of $|\chi\rangle$ relative to Alice's agent-situation at $A{ }^{7}$ Then Alice at $A$ should not assign state $|\chi\rangle$ relative to her agent-situation, while correctly maintaining that her Qbit should be assigned some initial pure state, relative to a different agent-situation such as Carol's just after implementing her unitary transformation.

In fact Bob at $B$ is in a similar position. The existence of the specific physical channel required for reliable teleportation does not guarantee the existence of any other physical channel capable of giving access at $B$ to conditions $K$ that back assignment of initial state $|\chi\rangle$ to Alice's Qbit relative to her agent-situation at $A$. If those conditions are physically inaccessible from $B$ then they do not back assignment of initial state $|\chi\rangle$ to Alice's Qbit relative to Bob's agent-situation at $B$. In that case Bob at $B$ should not assign a state to Alice's Qbit, while correctly maintaining that her Qbit should be assigned some initial pure state, relative to a different agent-situation such as Alice's or Carol's.

There is even a scenario in which initial pure state $|\chi\rangle$ correctly assigned to Alice's Qbit by Carol (relative to her agent-situation just after preparing it) is unknown to Alice but known to Bob. This would involve the presence of physical channels rendering the conditions $K^{\prime}$ accessible from Bob's agent-situation at $B$ but the absence of physical channels rendering conditions $K^{\prime}$ accessible from Alice's agent-situation at $A$. In this scenario a quantum state unknown to Alice could be known to Bob whether or not it was teleported from Alice to Bob!

To sum up, conditions in the past light cone of an agent-situation $G$ may back assignment of a quantum state to a system relative to some other agent-situation $H$, without backing assignment of a quantum state relative to $G$. Alice and/or Bob may each be in such an agent-situation in a case of quantum teleportation. That is how it can make sense to speak of teleporting an unknown quantum state: it makes sense when the state correctly assigned relative to Carol's agent-situation is unknown to Alice and/or Bob, at least one of whom is in an agent-situation relative to which no quantum state assignment is possible. Since an agent-situation need not be occupied by any actual agent, Carol's presence is not required.

\section{Conclusion}

A quantum state functions as an informational bridge between its backing conditions and its advice conditions. Since it is assigned from the perspective of a physically-characterized agent-situation, what quantum state is assigned is relative to that agent-situation. But this does not make quantum state assignments subjective or dependent on the epistemic state of any agent. Quantum state assignments are objectively correct or incorrect: only correct state assignments are reliable informational bridges. What makes them reliable are the patterns of

\footnotetext{
${ }^{7}$ But doesn't Carol herself provide such a physical channel? Not if all traces of what she did have been erased from her brain and the rest of her body.
} 
statistical correlation between their backing conditions and their advice conditions that obtain in the physical world. There are objectively correct quantum state assignments whether or not there are any agents capable of making them. But it is physically situated, and so epistemically limited, agents who naturally take the perspective of an agent-situation and so find it useful to assign quantum states from that perspective.

Are quantum states real? If they are, what do they represent? There are real patterns of statistical correlation in the physical world. Correctly assigned quantum states reliably track these through legitimate applications of the Born rule. But the Born rule does not directly describe these patterns: the frequencies they display are not in exact conformity to that rule - the unexpected does happen. A quantum state is real in so far as it specifies the real probabilistic relations between its backing conditions and its advice conditions. Those probabilistic relations are objective. What they are depends on conditions in the physical world, even though probability statements do not directly describe those conditions. So a quantum state is not an element of physical reality (it is not a beable): $|\psi\rangle$ does not represent a physical system or its properties. But it is real nonetheless. The world contains quantum states just as it contains probabilities, physicists and their journals. 


\section{References}

[1] Bell, J.S. [2004]: Speakable and Unspeakable in Quantum Mechanics. Revised edition. Cambridge: Cambridge University Press.

[2] Brun, T., Finkelstein, J. and Mermin, N.D. [2002]: "How much state assignments can differ", Physical Review A, 65 (2002), 032315.

[3] Egg, M. [2013]: "Delayed-choice experiments and the metaphysics of entanglement", Foundations of Physics, 43:1124-1135.

[4] Gleason, A.M. [1957]: "Measures on the closed subspaces of a Hilbert space", Journal of Mathematics and Mechanics, 6: 885-93.

[5] Healey, R.A. [2012a]: "Quantum theory: a pragmatist approach", British Journal for the Philosophy of Science, 63: 729-71.

[6] Healey, R.A. [2012b]: "Quantum decoherence in a pragmatist view", Foundations of Physics, 42: 1534-55.

[7] Healey, R.A. [forthcoming]: "Locality, probability and causality", in Quantum Nonlocality and Reality, Mary Bell and Gao Shan, eds. Cambridge: Cambridge University Press.

[8] Leifer, M. [2014]: "Is the quantum state real?", Quanta, 3: 67-155.

[9] Ma, X-S. [2012]: "Experimental delayed-choice entanglement swapping", Nature Physics, 8: 480-485.

[10] Maudlin, T. [2011]: Quantum Non-Locality and Relativity, 3rd edition. Chichester, West Sussex: Wiley-Blackwell.

[11] Mermin, N.D. [2002]: "Whose knowledge?", in R.A. Bertlmann and A. Zeilinger eds. Quantum [Un]Speakables. Berlin: Springer Verlag.

[12] Mermin, N.D. [2007]: Quantum Computer Science. Cambridge: Cambridge University Press.

[13] Peres, A. [2000]: "Delayed-choice for entanglement swapping", Journal of Modern Optics, 47.

[14] Price, H. [1996]: Time's Arrow and Archimedes' Point. Oxford: Oxford University Press.

[15] Timpson, C.G. [2013]: Quantum Information Theory. Oxford: Clarendon Press.

[16] Weihs, G., Jennewein, T., Simon, C., Weinfurter, H., and Zeilinger, A. [1998]: "Violation of Bell's inequality under strict Einstein locality conditions", Phyiscal Review Letters, 81: 5039-5043. 